\title{
APLICAÇÃO DA METODOLOGIA GDE/UNB EM UM VIADUTO: ESTUDO DE CASO NO MUNICÍPIO DE CURVELO-MG
}

\section{GDE/UNB METHODOLOGY APPLICATION A VIADUCT: CASE STUDY IN CURVELO-MG}

\author{
GONÇALVES FILHO, EDSON \\ Estudante de graduação \\ CEFET-MG - Curvelo \\ Minas Gerais; Brasil \\ edson.gonfilho@gmail.com
}

PENA BORTONE, THIAGO

Professor EBTT

CEFET-MG - Curvelo

Minas Gerais; Brasil

thiago.bortone@cefetmg.br

JARDIM MARTINI, RACHEL

Professora EBTT

CEFET/MG

Minas Gerais; Brasil

martini@cefetmg.br

\author{
GREGÓRIO PACHECO, SÍLVIA \\ Estudante de graduação \\ CEFET-MG - Curvelo \\ Minas Gerais; Brasil \\ silvia.gpacheco@hotmail.com \\ DE PAULO RAMOS, MARCOS \\ Professora EBTT \\ CEFET-MG - Curvelo \\ Minas Gerais; Brasil \\ marcos.ramos@cefetmg.br
}

\author{
PATRÍCIA FERREIRA, LUCIANA \\ Professora EBTT \\ CEFET-MG - Curvelo \\ Minas Gerais; Brasil \\ lupiferreira@cefetmg.br
}

\section{RESUMO}

As Obras de Arte Especiais (OAE's) são construções de engenharia com características estruturais, construtivas e funcionais específicas e requerem habilidades técnicas para seu projeto, execução e manutenção. Toda estrutura em concreto armado deteriora e sua vida útil especificada em projeto depende da realização de manutenções adequadas e em tempo certo, a fim de se garantirem os requisitos de funcionalidade, segurança, estética, economia e durabilidade. Nesse sentido, a metodologia Grau de Deterioração da Estrutura da Universidade de Brasília (GDE/UnB), adaptada por Verly (2015), visa identificar os problemas da estrutura, sistematizar um processo de inspeção e categorizar os sistemas estruturais de acordo com a gravidade das manifestações patológicas verificadas. Este trabalho tem por objetivo avaliar condição estrutural de uma OAE, a partir da aplicação da metodologia GDE/UnB, por meio de um estudo de caso no Município de Curvelo/MG. Os resultados mostram que o critério de avaliação utilizado é adequado em inspeção de pontes rodoviárias para fins de gestão de manutenções.

Palavras-chave: durabilidade, manutenção, inspeção, critério de avaliação.

\begin{abstract}
Bridges and tunnels are engineering constructions with specific structural, constructive and functional characteristics and require technical skills for their design, execution and maintenance. Every reinforced concrete structure deteriorates and its design-specified service life depends on proper and timely maintenance to meet the requirements of functionality, safety, aesthetics, economy and durability. In the sense, Degree of Deterioration of Structure methodology of the University of Brasilia (GDE/UnB), adapted by Verly (2015), aims to identify the structure problems, systematize an inspection process and categorize the structural systems according to the severity of the structural pathologies. This paper aims to evaluate the structural condition of a highway bridge from the application of the GDE/UnB methodology through a case study in Curvelo/MG. The results show that the evaluation criterion used is adequate in highway bridge inspection for maintenance management purposes.

Keywords: durability, maintenance, inspection, evaluation criteria.
\end{abstract}




\section{INTRODUÇÃO}

As Obras de Arte Especiais (OAE's) têm papel importante no desenvolvimento econômico do país, ao permitir o deslocamento de pessoas e mercadorias em trechos logisticamente mais eficientes. São construções de engenharia com características estruturais, construtivas e funcionais que requerem habilidades técnicas específicas e criativas para seu projeto, execução e manutenção (MITRE, 2005).

Contudo, as OAE's, como todo sistema estrutural, sofrem deterioração de seus materiais ao longo do tempo, o que pode implicar na alteração de seu comportamento, sendo possível, inclusive, a perda do desempenho estrutural proposto inicialmente em projeto (GOMES; XAVIER; FRANCISCO, 2018).

De acordo com Verly (2015), tanto a interdição ou restrição de carga em uma determinada OAE implica em transtornos ao usuário da via. Dependendo do tipo de carga transportada, atrasos podem implicar na sua completa inutilização.

Conforme observa Vitório (2013), as OAE's são mais vulneráveis uma vez que, geralmente, se localizam em ambientes de forte agressividade ambiental que podem proporcionar desequilíbrios associados às manifestações patológicas que atacam as estruturas trazendo graves consequências à vida útil e à funcionalidade das mesmas. Isso justifica a importância de se ter inspeções periódicas a fim de que sejam efetuadas manutenções em tempo adequado para garantir à OAE a vida útil prevista em projeto. A NBR 6118 (ABNT, 2014) recomenda que os projetos das estruturas em concreto sejam construídos de forma que sejam facilitadas as inspeções e manutenções preventivas da construção.

Verly (2015) afirma que a avaliação de pontes ou viadutos pode ser feita em partes ou da estrutura como um todo, desde que se baseie em dados coletados em inspeções. Giovannetti (2014) define inspeção como um conjunto de procedimentos técnicos realizados com o objetivo de definir as características de uma obra em um instante específico. A inspeção pode ser visual ou feita através de ensaios e medições com uso de equipamentos. A inspeção visual possui melhor relação custo/informação, a depender de profissional qualificado, tornando-se ferramenta eficiente para se acompanhar a evolução de manifestações patológicas em uma estrutura (VERLY, 2015).

Atualmente, há variadas metodologias para inspeção de pontes e viadutos. São exemplos: o método da NBR 9452 Inspeção de pontes, viadutos e passarelas de concreto - Procedimento (ABNT, 2019), a Norma 010-PRO - Inspeções em pontes e viadutos de concreto armado e protendido - Procedimento (DNIT, 2004), e o método Grau de Deterioração da Estrutura (GDE) da Universidade de Brasília (UnB). Na metodologia GDE/UnB busca-se associar cada manifestação patológica constatada a um determinado valor, de acordo com o elemento onde a anomalia se encontra e sua intensidade e, dessa forma, a partir de equações específicas da metodologia, atribuir uma nota quantitativa e objetiva à OAE em análise.

Este trabalho analisa e aplica a metodologia GDE/UnB adaptada por Verly (2015) para a inspeção de pontes e viadutos. A finalidade é avaliar a condição estrutural e verificar a adequação dessa metodologia em uma OAE situada no Município de Curvelo/MG.

\section{METODOLOGIA GDE EM PONTES RODOVIÁRIAS}

De acordo com Verly (2015), a metodologia GDE/UnB surgiu a partir de um método utilizado em 1991 pela Universidade Federal do Rio Grande do Sul (UFRGS) para inspecionar OAEs para a Prefeitura Municipal de Porto Alegre/RS (KLEIN apud VERLY, 2015). A metodologia se baseava em identificar os problemas da estrutura, sistematizar um processo de inspeção e categorizar os sistemas estruturais de acordo com a gravidade das manifestações patológicas verificadas.

Conforme relata Verly (2015), a partir do trabalho da UFRGS, Castro (1994) desenvolveu uma metodologia para avaliar as estruturas de concreto em edificações de diferentes ocupações. O método foi posteriormente adaptado por Lopes (1998), para edifícios comerciais do Banco do Brasil; por Boldo (2002), em quarenta edificações do Exército Brasileiro; por Fonseca (2007), para avaliação de estruturas de concreto em edificações, e por Euqueres (2011), que avaliou onze pontes no estado de Goiás. Posteriormente, a metodologia foi adaptada por Verly (2015) em avaliação de OAE's.

Na metodologia GDE/UnB é necessário separar os componentes de uma OAE em famílias de elementos típicos e para cada elemento de uma família deve se ponderar um fator de dano e atribuir um fator de intensidade do dano. A partir 
desses fatores, calcula-se o grau de dano equivalente aos fatores atribuídos, o grau de deterioração do elemento, o grau de deterioração da família de elementos e, após introduzir um fator de relevância dessa família, calcular o grau de deterioração da estrutura.

\subsection{Divisão em famílias de elementos típicos}

Para a aplicação da metodologia GDE/UnB é necessário que os elementos componentes da OAE sejam agrupados de acordo com suas características e funções estruturais. Deve-se atentar quando um mesmo elemento possui funções diferentes, como é o caso da transversina que pode ser portante ou de ligação. A transversina portante suporta o carregamento permanente de uma laje e atua na distribuição transversal de esforços (VERLY, 2015). Já a transversina de ligação atua apenas na distribuição dos esforços. Cada família recebe um fator de relevância estrutural $\left(\mathrm{F}_{\mathrm{r}}\right)$.

\subsection{Fator de ponderação do dano $\left(F_{p}\right)$}

O fator de ponderação do dano, como descreve Verly (2015), é a forma de relacionar o dano observado com as condições de segurança, funcionalidade e estética dos elementos de uma determinada família. A partir disso, uma mesma manifestação patológica pode possuir fatores de ponderação diferentes, a depender de onde está localizada.

\subsection{Fator de intensidade do dano $\left(F_{i}\right)$}

O objetivo do fator de intensidade do dano é atribuir valores correspondentes à gravidade e evolução do dano em um determinado elemento. Varia de 0 a 4 , sendo 0 elementos sem lesões a 4 elementos em estado crítico. Para este fator, é importante observar a profundidade do dano, ambiente onde o dano se encontra e deformações aparentes.

\subsection{Grau do dano (D)}

O grau do dano $(D)$ é fator que relaciona o fator de intensidade e o fator de ponderação a partir das Equações 01 e 02.

$$
\begin{array}{cr}
D=0,8 \cdot F i \cdot F p & \text { Para } F i \leq 2,0 \\
D=(12 \cdot F i-28) F p & \text { Para } F i \geq 3,0
\end{array}
$$

\subsection{Grau de deterioração do elemento $\left(G_{\text {de }}\right)$}

A partir da Equação 03 é possível analisar os efeitos dos danos no elemento. Essa equação foi proposta por Lopes (1998) a fim de que aos efeitos do maior dano sejam adicionados os efeitos dos demais.

$$
G \quad\left[1 \frac{\left(\sum\right.}{\Sigma}\right]
$$

Onde:

$\mathrm{D}_{\mathrm{i}}$ : grau do dano de índice "i”;

$\mathrm{D}_{\text {máx }}$ : maior grau do dano encontrado no elemento;

$\mathrm{G}_{\mathrm{de}}$ : grau de deterioração do elemento;

n: número de danos encontrados no elemento.

A partir do valor encontrado na equação 03 têm-se o nível de deterioração e ações a serem recomendadas, conforme Tabela 1.

Tabela 1 - Nível de Deterioração.

\begin{tabular}{c|c|l}
\hline Nível de deterioração & $\mathrm{G}_{\mathrm{de}}$ & \multicolumn{1}{c}{ Ações a serem adotadas } \\
\hline Baixo & $0-15$ & Estado aceitável. Manutenção preventiva. \\
\hline Médio & $15-50$ & $\begin{array}{l}\text { Definir prazo/natureza para nova inspeção. Planejar intervenção } \\
\text { em longo prazo (máximo 2 anos). }\end{array}$ \\
\hline Alto & $50-80$ & $\begin{array}{l}\text { Definir prazo/natureza para inspeção especializada detalhada. } \\
\text { Planejar intervenção em médio prazo (máximo 1 ano). }\end{array}$ \\
\hline Sofrível & $80-100$ & $\begin{array}{l}\text { Definir prazo-/natureza para inspeção especializada detalhada. } \\
\text { Planejar intervenção em curto prazo (máximo 6 meses). }\end{array}$ \\
\hline Crítico & $>100$ & Inspeção especial emergencial. Planejar intervenção imediata. \\
\hline
\end{tabular}


Fonte: Verly, 2015.

\subsection{Grau de deterioração de uma família de elementos $\left(G_{d f}\right)$}

$O \mathrm{G}_{\mathrm{df}}$ apresenta o grau de deterioração dos elementos pertencentes a uma mesma família. Para tanto, utiliza os valores de Gde de cada elemento da família que está sendo analisada (Equação 04).

$$
\sqrt{1 \frac{\left(\sum\right.}{\sum}}
$$

Onde:

$\mathrm{G}_{\mathrm{df}}$ : grau de deterioração de uma família de elementos;

$\mathrm{G}_{\mathrm{de}, \max }$ : Maior grau de deterioração encontrado na família de elementos

$\mathrm{G}_{\mathrm{de}, \mathrm{i}}:$ Graus de deterioração apresentados pelos elementos da família $(\geq 15)$;

$\mathrm{m}$ : número de elementos com $\mathrm{G}_{\mathrm{de}} \geq 15$.

\subsection{Fator de relevância estrutural da família $\left(\mathbf{F}_{\mathrm{r}}\right)$}

O Fr tem por objetivo graduar a importância da família de elementos análise no comportamento e no desempenho do sistema estrutural da OAE avaliada.

Tabela 2 - Fator de relevância de acordo com a família de elementos.

\begin{tabular}{l|c}
\hline \multicolumn{1}{c|}{ Família } & Fr \\
\hline Barreiras, guarda-corpo, guarda rodas, pista de rolamento. & 1 \\
\hline Juntas de dilatação & 2 \\
\hline Transversinas, cortinas, alas & 3 \\
\hline Lajes, fundações vigas secundárias, aparelhos de apoio & 4 \\
\hline Vigas e pilares principais & 5 \\
\hline
\end{tabular}

Fonte: Verly, 2015.

\subsection{Grau de deterioração da estrutura $\left(G_{d, \text { mod }}\right)$}

Verly (2015) apresenta a Equação 05 como alternativa para cálculo do grau de deterioração da estrutura modificado $\left(\mathrm{G}_{\mathrm{d}, \mathrm{mod}}\right)$.

$$
-\sqrt{1 \frac{\left(\sum\right.}{\sum}}
$$

Onde:

$\mathrm{G}_{\mathrm{d} \text {,mod}}:$ grau de deterioração da estrutura modificado;

$\mathrm{k}$ : número de famílias da estrutura;

$\mathrm{K}$ : produto do $\mathrm{G}_{\mathrm{df}, \mathrm{i}}$ pelo respectivo $\mathrm{F}_{\mathrm{r}}$;

$\mathrm{K}_{\text {máx }}$ : maior valor do produto do $\mathrm{G}_{\mathrm{df}, \mathrm{i}}$ pelo respectivo $\mathrm{F}_{\mathrm{r}}$.

\section{ESTUDO DE CASO}

O presente estudo de caso é referente à inspeção de uma OAE situada na rodovia CMG-259, sobre a BR-135, no Município de Curvelo/MG, coordenadas geográficas $18^{\circ} 44^{\prime} 50.28^{\prime \prime} \mathrm{S}$ e $44^{\circ} 26^{\prime} 45.75^{\prime \prime} \mathrm{O}$ (Figuras 1 e 2). O viaduto possibilita a continuidade da rodovia CMG-259 que liga a cidade de João Neiva/ES à BR-040 no Município de Felixlândia/MG. A OAE está localizada dentro do perímetro urbano do Município de Curvelo/MG e é o acesso principal ao bairro Santa Rita, onde se situa o Centro Federal de Educação Tecnológica de Minas Gerais (CEFET-MG). 


\section{CBPAT 2020 \\ CONGRESSO BRASILEIRO DE PATOLOGIA DAS CONSTRUÇÕES \\ DE 15 A 17 DE ABRIL | FORTALEZA - CE \\ ANAIS}

ISBN 978-65-86819-05-2 ASSOCIACCĀO BRASILEIRA CONSTRUÇOES

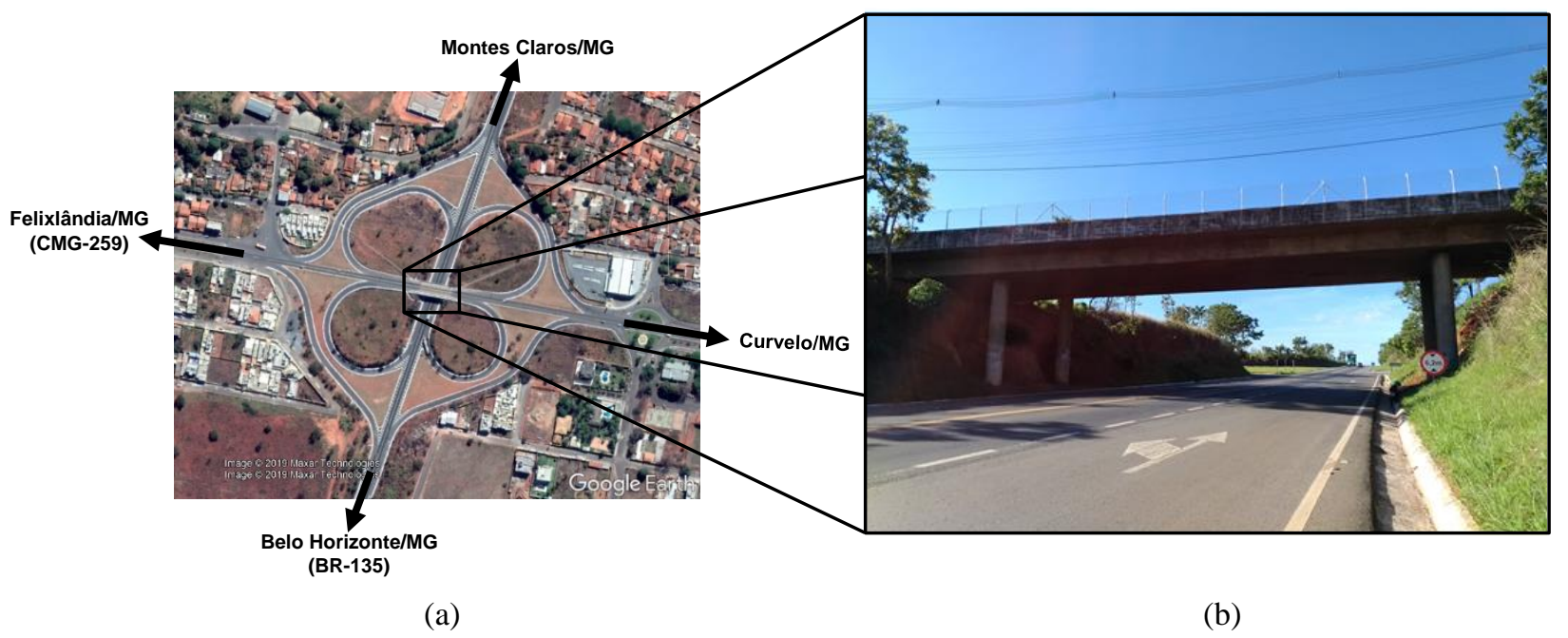

Figura 1: Localização da OAE em estudo. (a) Imagem de satélite. (b) Vista frontal. Fonte: Esquerda - adaptada Google Earth (2019) e direita - autores.

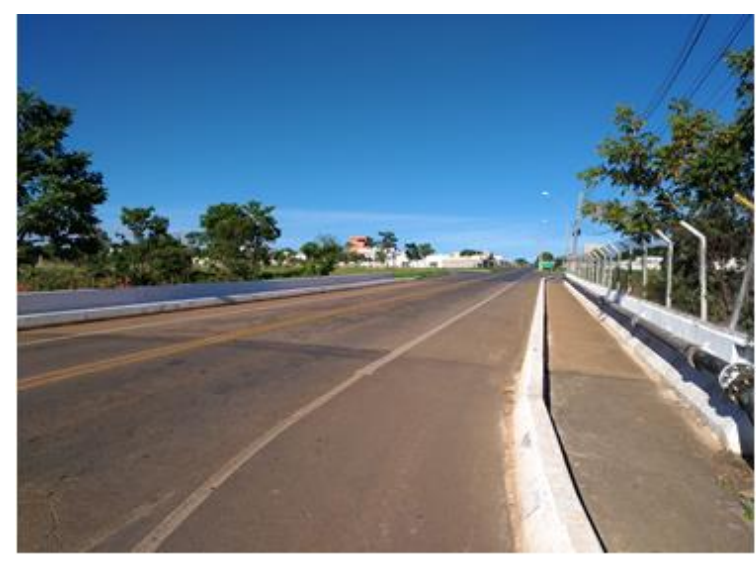

(a)

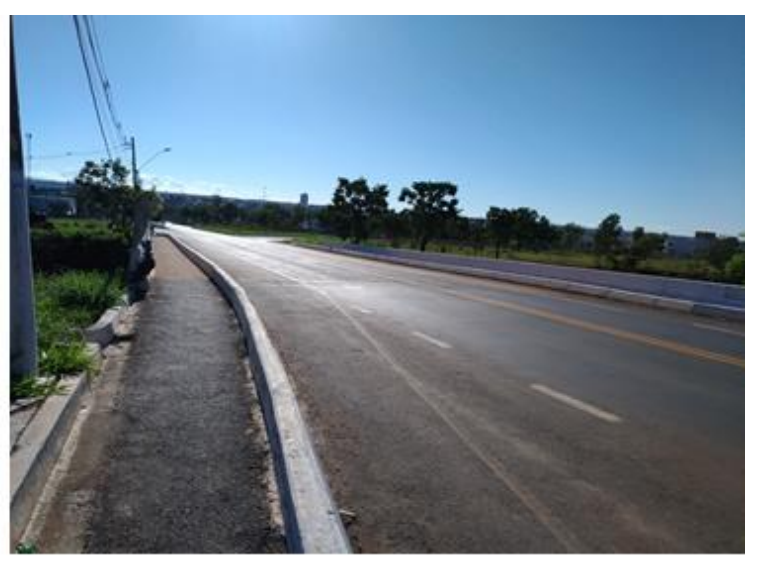

(b)

Figura 2: Caracterização do viaduto. (a) Vista sentido Curvelo-Felixlândia. (b) Vista sentido Felixlândia-Curvelo. Fonte: Autores.

Esta OAE trata-se de viaduto do tipo caixão (modelo de laje alveolar), em concreto armado e moldado in loco, com geometria horizontal em tangente, extremidades em balanço e esconsidade de aproximadamente $12^{\circ}$ em relação ao eixo da rodovia CMG-259. A OAE possui, no sentido longitudinal, comprimento total de 40,40 m sendo um vão central de $24 \mathrm{~m}$ e dois balanços de 8,20 m e 7,20 m (Figura 3). No sentido transversal, possui largura total de $15 \mathrm{~m}$ com dois balanços de 2 m (Figura 4).

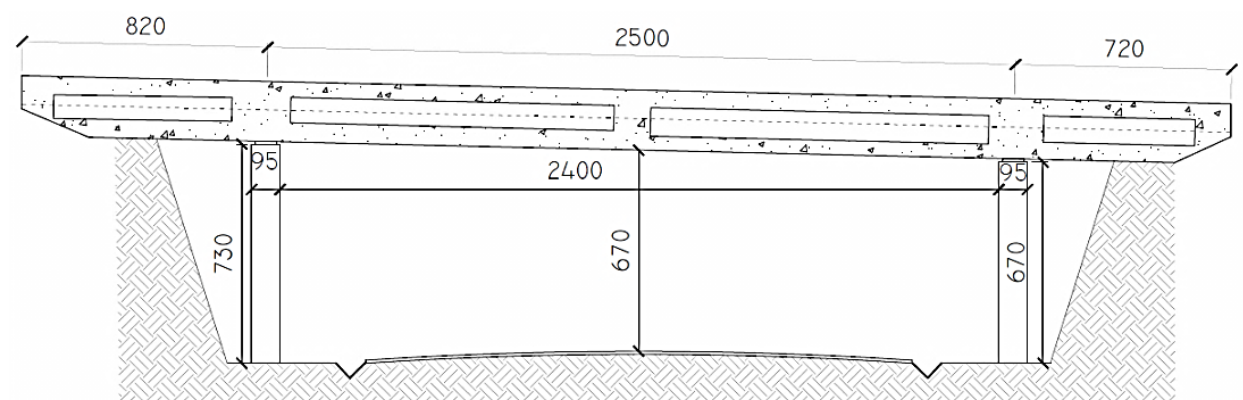

Figura 3: Corte longitudinal com dimensões aproximadas (sentido Belo Horizonte - Montes Claros) Fonte: Autores. 


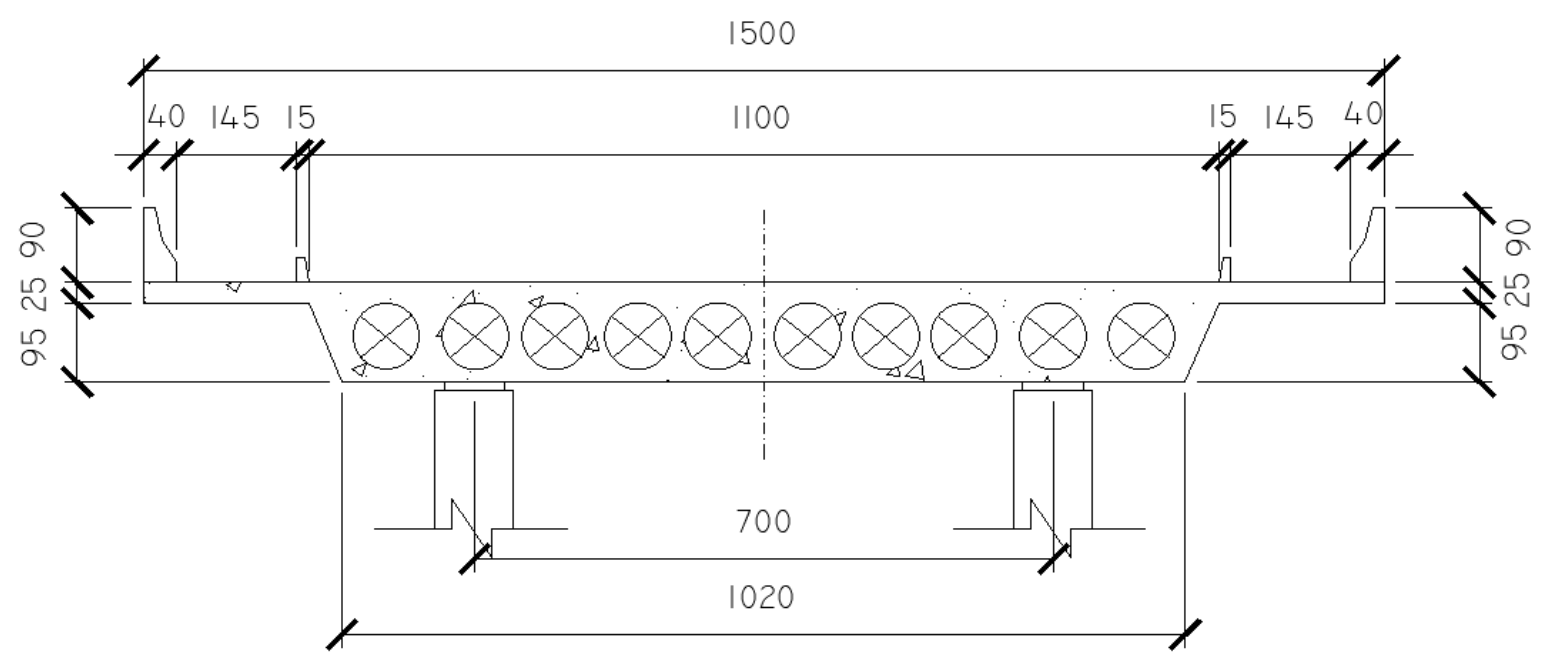

Figura 4: Corte transversal com dimensões aproximadas.

Fonte: Autores.

De forma geral, a OAE está em bom estado de conservação, com talude íngreme e contenção do tipo rip-rap. Contudo, apresenta um processo de erosão no encabeçamento de ambos os lados, provocada possivelmente por águas pluviais (Figura 5), além da ausência de proteção dos pilares próximos à rodovia.

A infraestrutura não é visualmente avaliável, sem conter elementos da fundação expostos. A mesoestrutura e superestrutura possuem algumas manchas e fissuras, enquanto as cortinas, alas e aparelhos de apoio aparentam bom estado de conservação. Não foram identificadas caixas de inspeção para acesso ao interior da superestrutura.

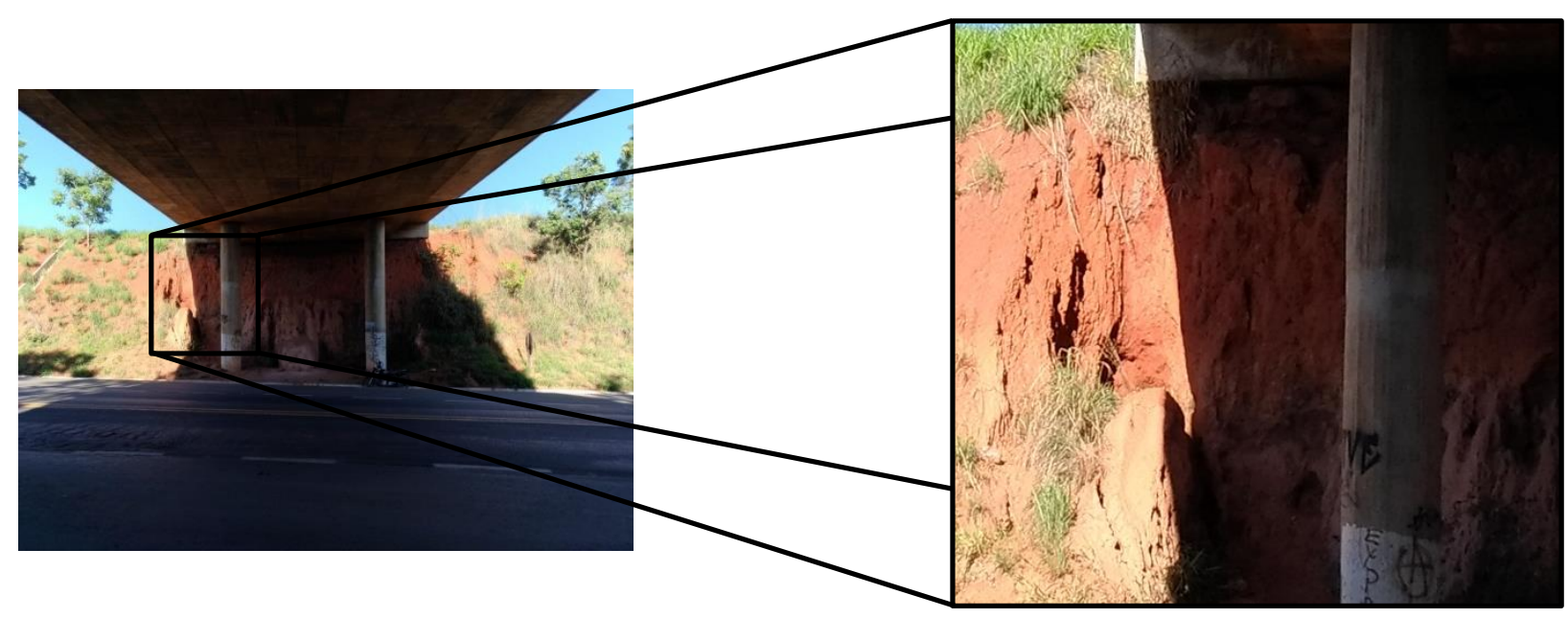

Figura 5: Processo de erosão no talude de encabeçamento da ponte.

Fonte: Autores.

\section{METODOLOGIA}

A inspeção foi realizada de forma visual, sendo utilizadas trena a laser, fita métrica, trena metálica e câmera fotográfica para caracterização da geometria dos elementos e registro dos fenômenos patológicos existentes. O diagnóstico se deu por etapas, sendo cada etapa feita para uma família de elementos típicos, conforme orienta a metodologia GDE/UnB. A metodologia GDE/UnB, revisada por Verly (2015) possui um documento de referência chamado de Manual de aplicação da metodologia $G D E / U n B$ em $O A E$ 's do qual foram retirados os fatores utilizados nessa caracterização. Após a identificação dos danos e atribuição dos fatores de intensidade foi possível o cálculo do valor numérico que representa o grau de deterioração da estrutura $\left(\mathrm{G}_{\mathrm{d}, \mathrm{mod}}\right)$. 


\section{CBPAT 2020 \\ CONGRESSO BRASILEIRO DE PATOLOGIA DAS CONSTRUÇÕES DE 15 A 17 DE ABRIL | FORTALEZA - CE \\ ISBN 978-65-86819-05-2 ASSOCIAC̄Á BRASILEIRA CONSTRUÇOES}

\subsection{Família A: Vigas e Pilares Principais}

O sistema estrutural que compõe a OAE é composto por dois pares de pilares de $0,95 \mathrm{~m}$ de diâmetro e altura próxima de 7 m, conforme Figura 6.

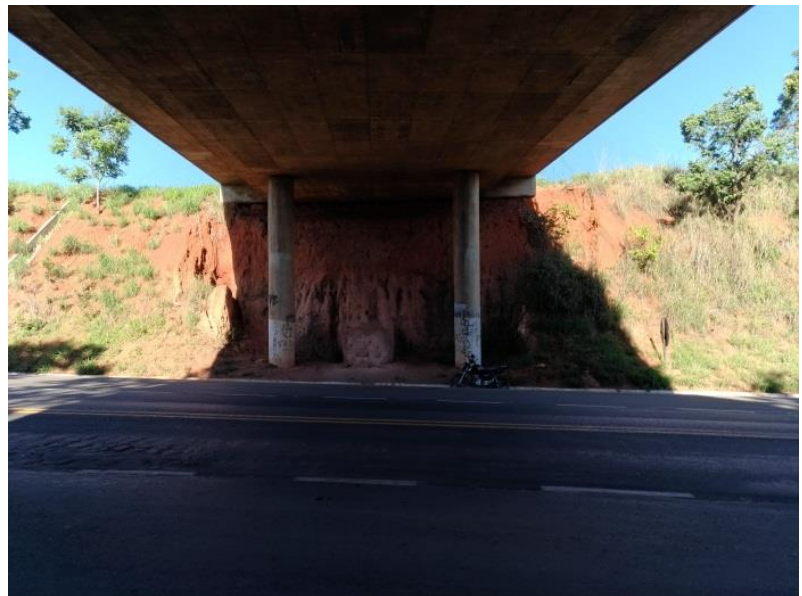

(a)

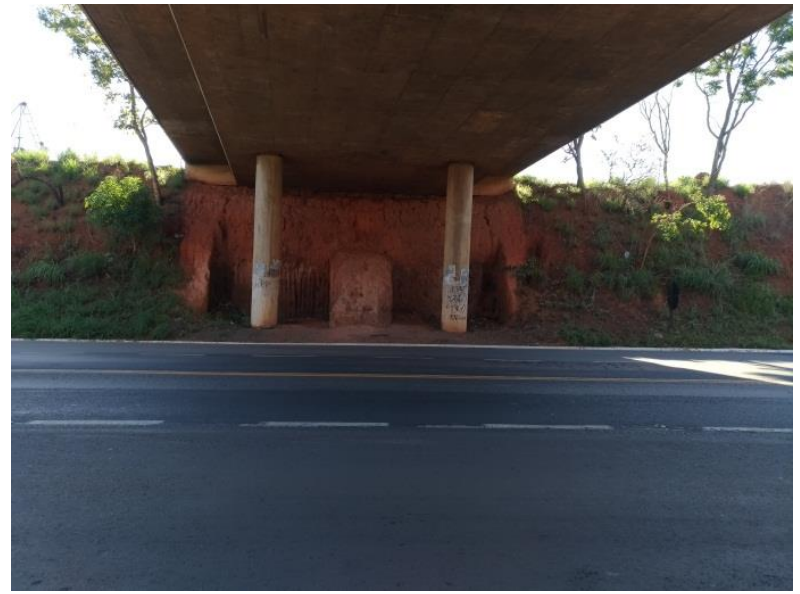

(b)

Figura 6: Vista frontal dos pilares. (a) Pilares lado esquerdo (sentido Montes Claros - Belo Horizonte). (b) Pilares lado direito (sentido Montes Claros - Belo Horizonte).

Fonte: Autores.

Foram identificadas falhas de concretagem em todos os pilares e pequenos desplacamentos em suas bases, com pontos de exposição da armadura do estribo e presença de corrosão (Figuras 7 e 8). Na metodologia GDE/UnB é sugerido um fator de ponderação igual a 3, tanto para falha de concretagem quanto desplacamentos em pilares, e igual a 5 para corrosão de armaduras. Para falha de concretagem, o fator de intensidade usado foi igual a 1, uma vez que é superficial e pouco significativa em relação às dimensões da peça. Já para o desplacamento, o fator de intensidade adotado foi igual a 2, por serem pequenas escamações do concreto. Para a corrosão será adotado o fator de intensidade igual a 2, uma vez que são manifestações leves, com pequenas manchas.

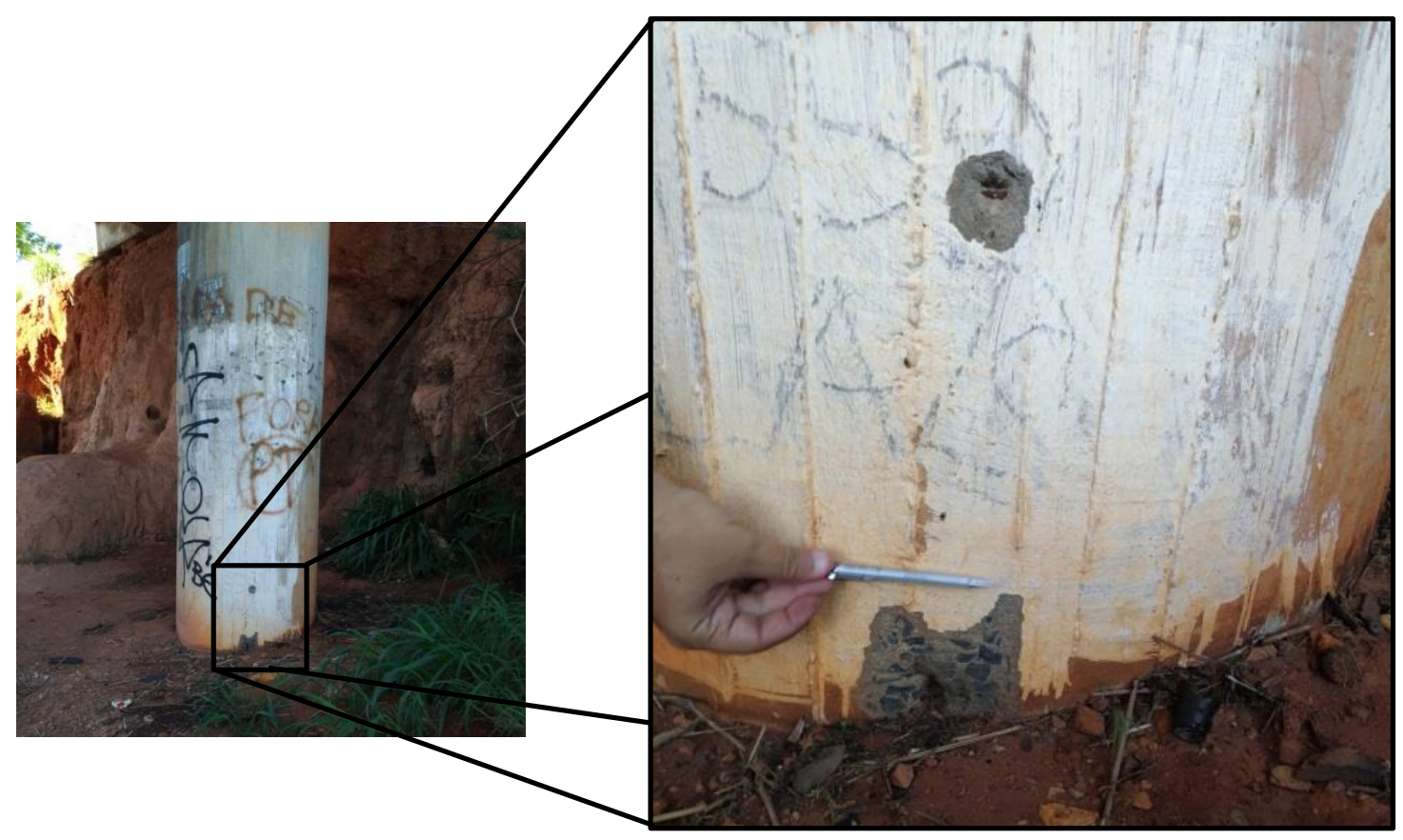

(a)

(b)

Figura 7: Desplacamento do concreto no pilar.

Fonte: Autores. 


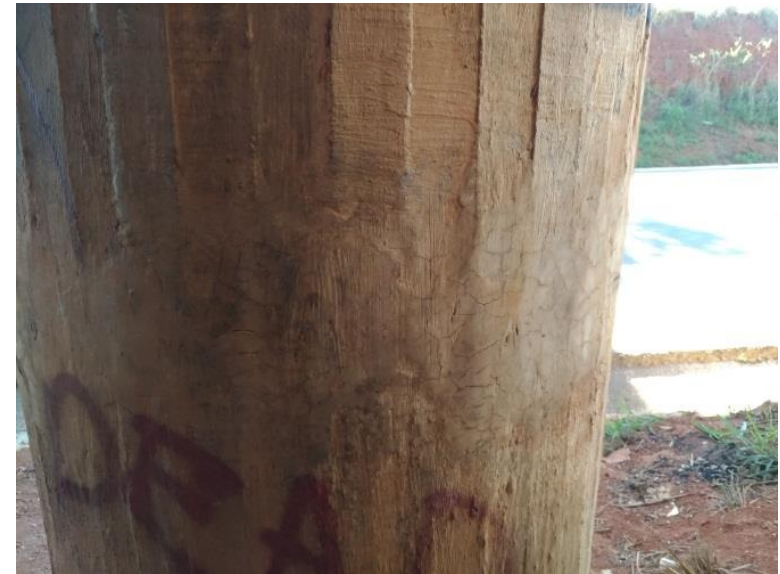

(a)

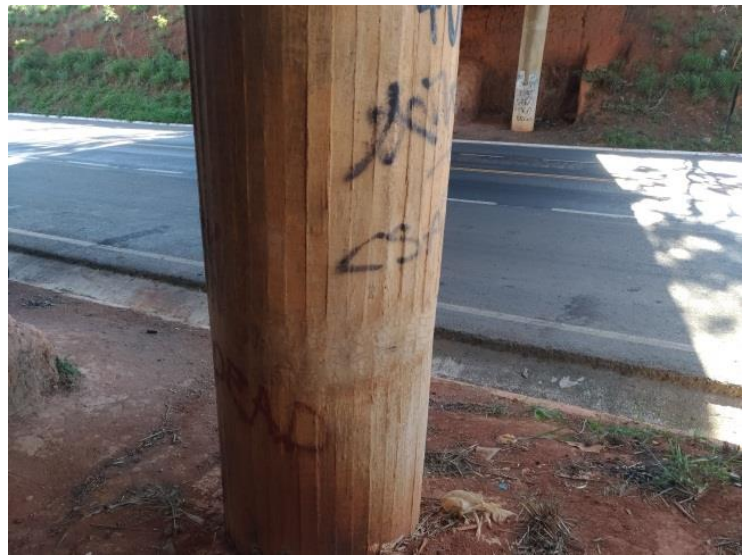

(b)

Figura 8: Falha de concretagem em pilar.

Fonte: Autores.

Considerando que se trata de um viaduto tipo caixão (modelo laje alveolar), não foi possível a identificação/vistoria das vigas.

\subsection{Família B: Lajes, fundações, vigas secundárias e aparelhos de apoio}

O viaduto em análise é composto por uma laje inferior (abaixo dos alvéolos) e uma laje superior (acima dos alvéolos). A laje inferior apresentou carbonatação do concreto, fissuras e manchas (Figuras 9 e 10). O fator de ponderação sugerido pela metodologia utilizada é igual a 3 para carbonatação do concreto e manchas e igual a 2 para fissuras de abertura e extensão reduzidas. Já com relação ao fator de intensidade, foi adotado um fator igual a 2 para carbonatação do concreto por estar em ambiente seco e, provavelmente atingindo a armadura, enquanto para fissuras foi adotado o valor igual a 2 por aparentar estarem estabilizadas. Para as manchas, também foi adotado o fator de intensidade igual a 2 por serem de pequena extensão.

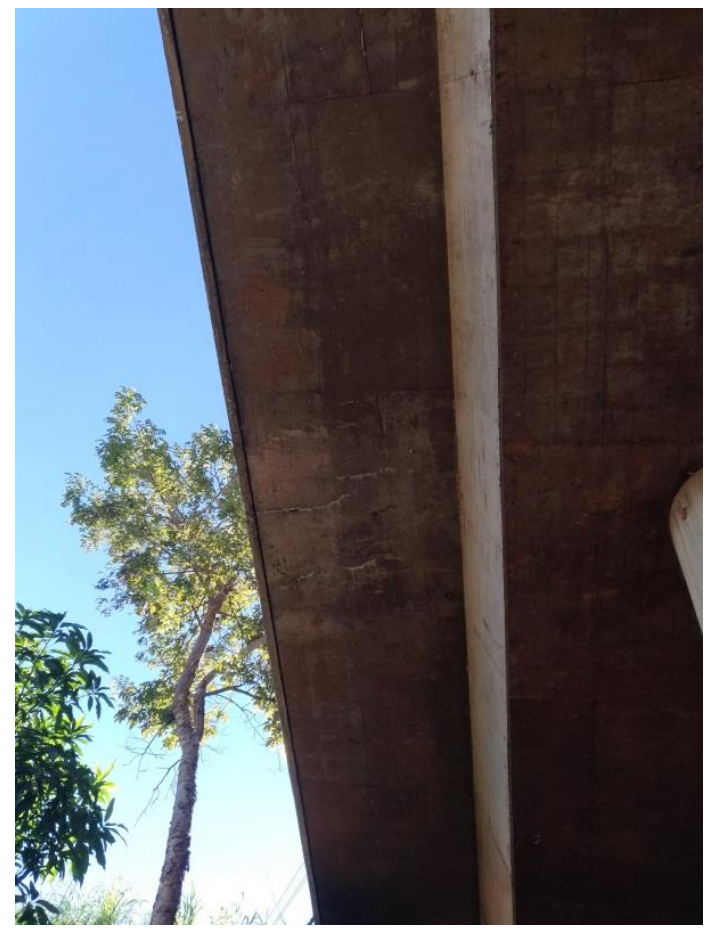

(a)

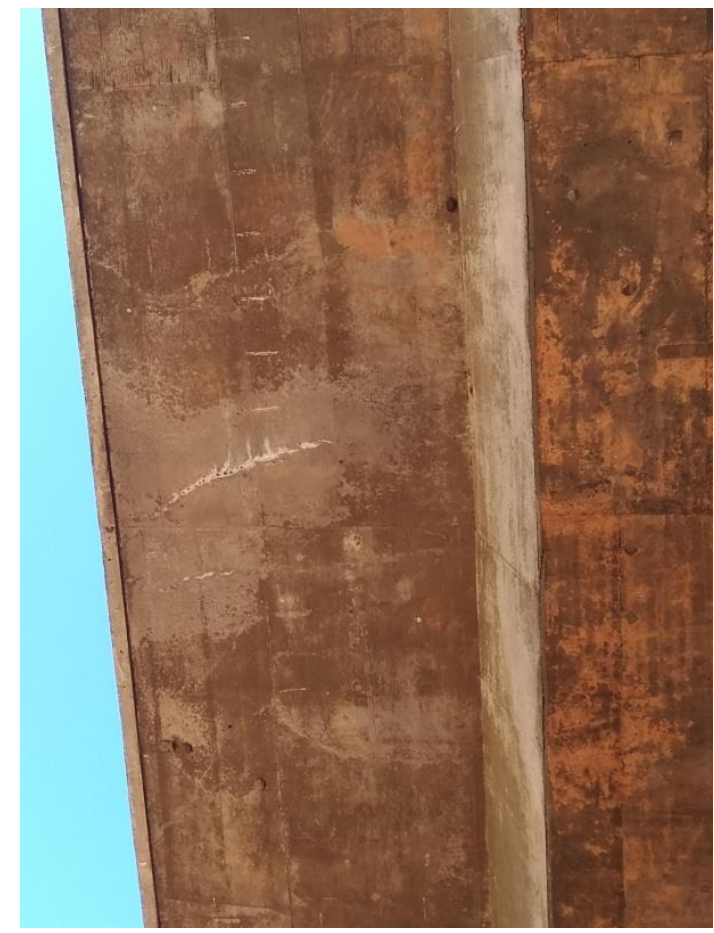

(b)

Figura 9: Carbonatação e fissuras nos balanços transversais do viaduto.

Fonte: Autores. 


\section{CBPAT 2020 \\ CONGRESSO BRASILEIRO DE PATOLOGIA DAS CONSTRUÇÕES \\ DE 15 A 17 DE ABRIL | FORTALEZA - CE

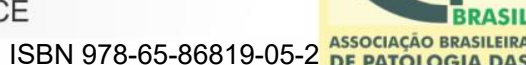 ASSOCIACÁO BRASILEIRA CONSTRUÇOES}

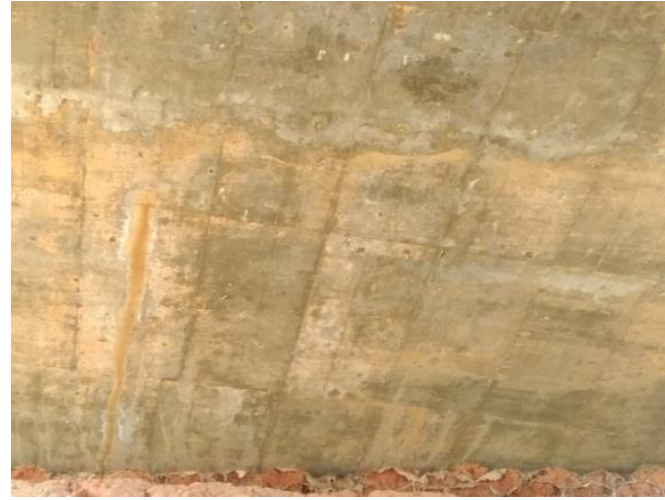

(a)

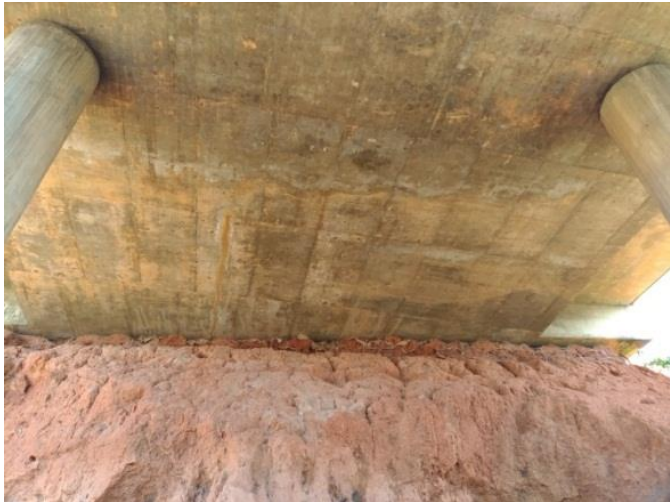

(b)

Figura 10: Manchas na laje inferior. Fonte: Autores.

A laje superior apresenta um cobrimento deficiente e fissuras, com armadura exposta e com corrosão em diversos pontos (Figuras 11 e 12). Para tanto, a metodologia sugere um fator de ponderação igual a 3 para cobrimento deficiente, quando esta se apresenta em lajes, igual a 2 para fissuras, de abertura e extensão reduzidas e iguais a 5 para corrosão das armaduras. Para o fator de intensidade, foi atribuído um valor igual a 3 para cobrimento deficiente, uma vez que possui armaduras expostas em extensões significativas. Para as fissuras foi adotado um valor igual a 2, por aparentar estarem estabilizadas. Já para a corrosão de armaduras foi atribuído um valor igual a 3, por ocorrem em dimensões significativas.

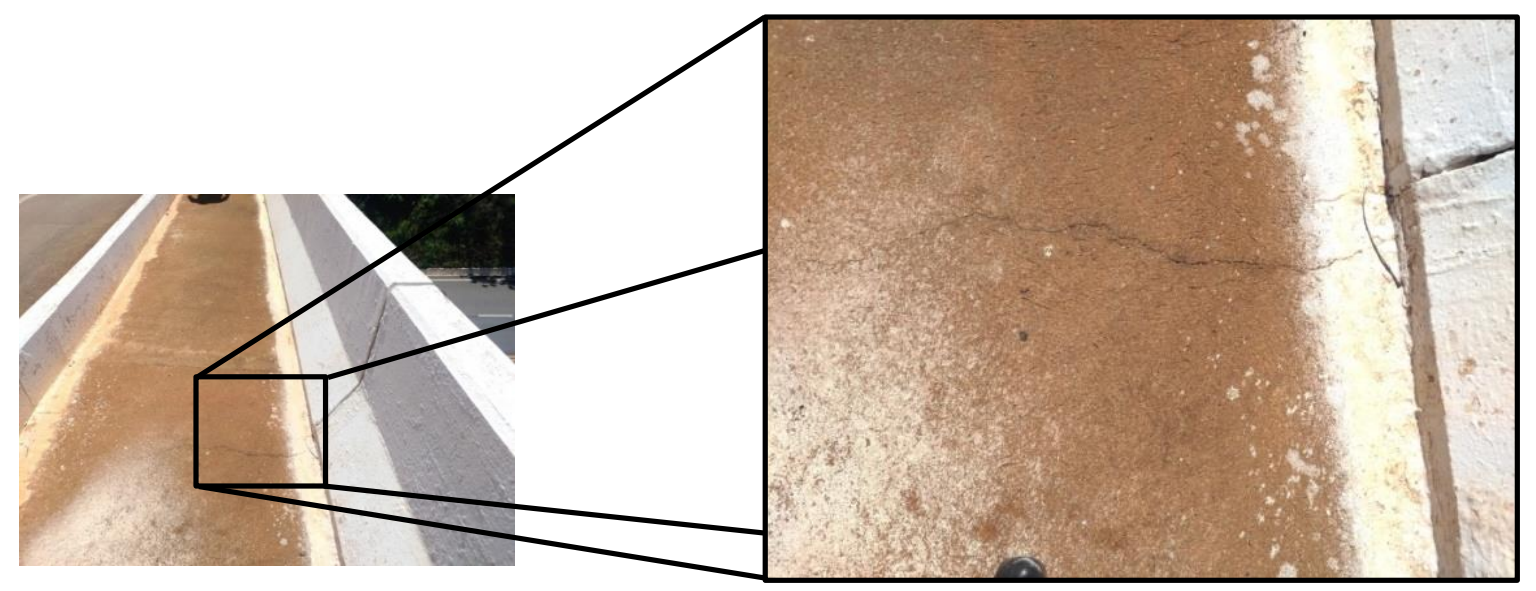

Figura 11: Fissuras na laje superior.

Fonte: Autores.

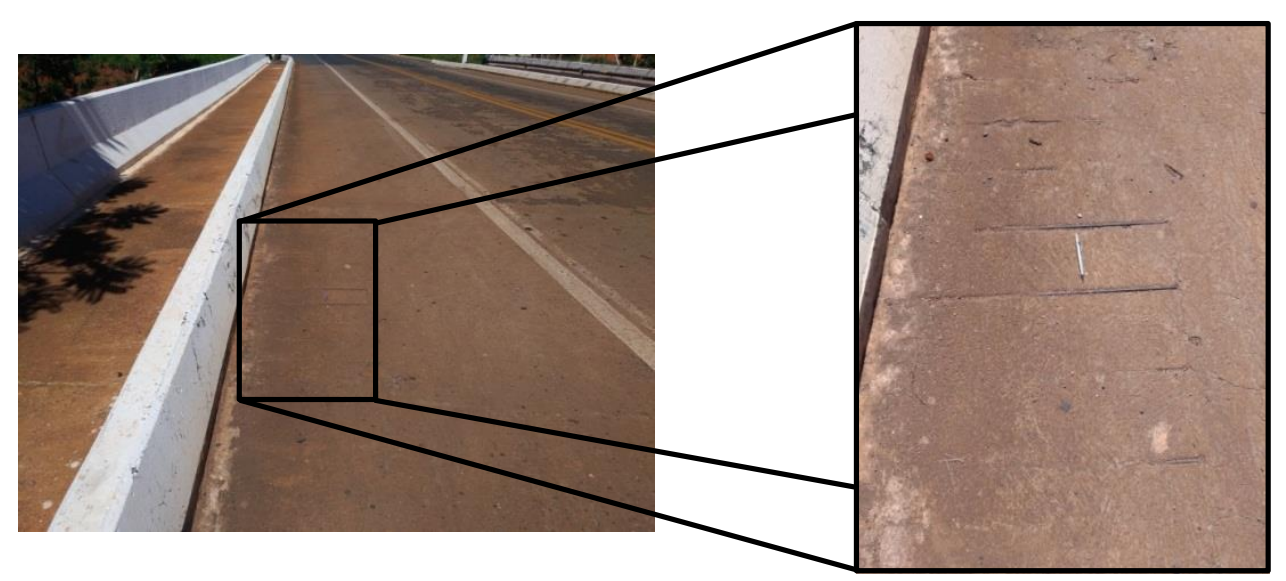

Figura 12: Cobrimento insuficiente com exposição da armadura. Fonte: Autores. 
As fundações e vigas secundárias não são visíveis, o que impossibilita a análise. Já os aparelhos de apoio aparentam estar em bom estado de conservação.

\subsection{Família C: Transversinas, cortinas, alas}

As transversinas não são visíveis para a inspeção. Já as cortinas e alas apresentam bom estado de conservação.

\subsection{Família D: Juntas de dilatação}

Não foram identificadas anomalias nas juntas de dilatação.

\subsection{Família E: Barreiras, guarda-corpo, guarda rodas, pista de rolamento}

As barreiras, guarda-corpo e guarda rodas apresentam bom estado de conservação, sem identificação de manifestações patológicas. Contudo a pista de rolamento possui desgaste superficial em estágio avançado (Figura 13). A metodologia GDE/UnB sugere um fator de ponderação igual a 5 para esta manifestação patológica e, considerando que há apresentação de armadura, foi atribuído o valor igual a 3 para o fator de intensidade.

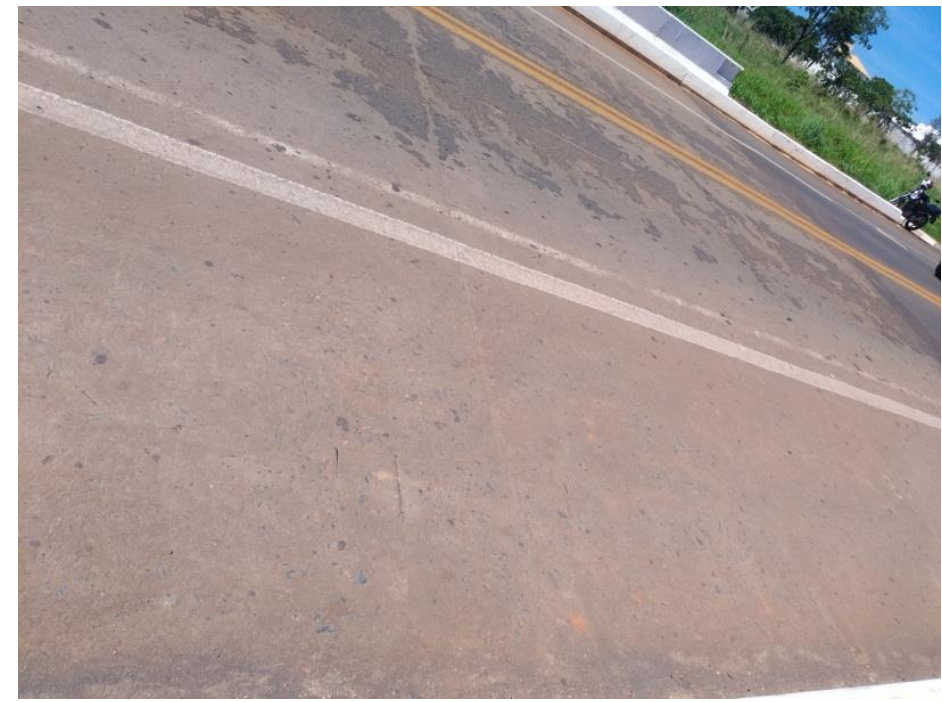

Figura 13: Desgaste superficial da pista de rolamento.

Fonte: Autores.

\section{RESULTADOS E DISCUSSÕES}

Para o cálculo do grau de deterioração da estrutura foram utilizados os valores de fator de relevância, dispostos na Tabela 2, de acordo com a família de elementos típicos. A partir dos fatores de ponderação e de intensidade especificados, foram possíveis os cálculos do grau de dano e grau de deterioração dos elementos, assim como o dano da família de elementos típicos, conforme equações de 1 a 4 . A partir da equação 5 foi possível o cálculo do grau de deterioração da estrutura. Os resultados obtidos e forma de cálculo estão dispostos na Tabela 4.

Conforme se observa a partir da Tabela 4, o grau de deterioração do viaduto é igual a 38,42 para a metodologia GDE/UnB. Isso mostra que, de acordo com a Tabela 1, se trata de um nível de deterioração médio. Isso posto, a partir da metodologia GDE/UnB, sugere-se uma intervenção na OAE em um prazo não superior a 2 anos, além de ser necessária nova inspeção em um espaço menor de tempo para acompanhamento da evolução das manifestações patológicas identificadas.

Verifica-se que as famílias que apresentaram maior dano foram a de barreiras, guarda-corpo, guarda rodas e pista de rolamento (devido ao desgaste superficial avançado) e a família de lajes, fundações, vigas secundárias e aparelhos de apoio, devido, principalmente, ao cobrimento insuficiente na laje superior. Supondo uma intervenção na OAE de recapeamento da mesa com consequente aumento do cobrimento nominal da armadura, em um segundo cálculo, 
constata-se que o nível de deterioração da estrutura passaria a ser 8,15, e classificar-se-ia como de nível baixo, estando em estado aceitável, sendo necessárias apenas manutenções preventivas.

Tabela 4 - Cálculo do grau de deterioração da estrutura.

\begin{tabular}{|c|c|c|c|c|c|c|c|c|c|}
\hline Família & $\mathbf{F r}$ & Elemento & $\begin{array}{l}\text { Manifestação } \\
\text { Patológica }\end{array}$ & $\mathbf{F p}$ & $\mathbf{F i}$ & $\begin{array}{l}\text { Grau de } \\
\text { dano }\end{array}$ & Gde & Gdf & $\mathbf{k}$ \\
\hline $\begin{array}{c}\text { Barreiras, } \\
\text { guarda-corpo, } \\
\text { guarda rodas, } \\
\text { pista de } \\
\text { rolamento } \\
\end{array}$ & 1 & $\begin{array}{l}\text { Pista de } \\
\text { rolamento }\end{array}$ & Desgaste superficial & 5 & 3 & 40 & 40 & 40,00 & 40,00 \\
\hline $\begin{array}{l}\text { Juntas de } \\
\text { dilatação }\end{array}$ & 2 & Juntas & Obstrução de junta & 5 & 2 & 8 & 8 & 8,00 & 16,00 \\
\hline $\begin{array}{c}\text { Transversinas, } \\
\text { cortinas, alas }\end{array}$ & 3 & - & - & - & 0 & - & 0 & 0,00 & 0,00 \\
\hline \multirow{6}{*}{$\begin{array}{l}\text { Lajes, fundações } \\
\text { vigas } \\
\text { secundárias, } \\
\text { aparelhos de } \\
\text { apoio }\end{array}$} & \multirow{6}{*}{4} & Laje inferior & $\begin{array}{l}\text { Carbonatação do } \\
\text { concreto }\end{array}$ & 3 & 2 & 4,8 & \multirow{3}{*}{7,8} & \multirow{6}{*}{59,52} & \multirow{6}{*}{238,07} \\
\hline & & Laje inferior & Fissuras & 2 & 2 & 3,2 & & & \\
\hline & & Laje inferior & Manchas & 3 & 2 & 4,8 & & & \\
\hline & & Laje superior & Cobrimento deficiente & 3 & 3 & 24 & \multirow{3}{*}{56,19} & & \\
\hline & & Laje superior & Fissuras & 2 & 2 & 3,2 & & & \\
\hline & & Laje superior & Corrosão & 5 & 3 & 40 & & & \\
\hline \multirow{12}{*}{$\begin{array}{c}\text { Vigas e pilares } \\
\text { principais }\end{array}$} & \multirow{12}{*}{5} & $\mathrm{P} 1$ & Desplacamento & 3 & 2 & 4,8 & \multirow{3}{*}{7,11} & \multirow{12}{*}{9,41} & \multirow{12}{*}{47,04} \\
\hline & & $\mathrm{P} 1$ & Falha de concretagem & 3 & 1 & 2,4 & & & \\
\hline & & $\mathrm{P} 1$ & Corrosão & 5 & 2 & 8 & & & \\
\hline & & $\mathrm{P} 2$ & Desplacamento & 3 & 2 & 4,8 & \multirow{3}{*}{7,11} & & \\
\hline & & $\mathrm{P} 2$ & Falha de concretagem & 3 & 1 & 2,4 & & & \\
\hline & & $\mathrm{P} 2$ & Corrosão & 5 & 2 & 8 & & & \\
\hline & & $\mathrm{P} 3$ & Desplacamento & 3 & 2 & 4,8 & \multirow{3}{*}{7,11} & & \\
\hline & & P3 & Falha de concretagem & 3 & 1 & 2,4 & & & \\
\hline & & P3 & Corrosão & 5 & 2 & 8 & & & \\
\hline & & $\mathrm{P} 4$ & Desplacamento & 3 & 2 & 4,8 & \multirow{3}{*}{7,11} & & \\
\hline & & $\mathrm{P} 4$ & Falha de concretagem & 3 & 1 & 2,4 & & & \\
\hline & & $\mathrm{P} 4$ & Corrosão & 5 & 2 & 8 & & & \\
\hline \multicolumn{9}{|c|}{ GRAU DE DETERIORAÇÃO DA ESTRUTURA (Gd,mod) } & $\mathbf{3 8 , 4 2}$ \\
\hline
\end{tabular}

Fonte: Autores.

\section{CONCLUSÕES}

A partir da aplicação da metodologia GDE/UnB no viaduto supracitado, verificou-se que este critério de avaliação é adequado na qualificação objetiva do estado de conservação e funcionamento de uma Obra de Arte Especial. Constatase ainda que esta metodologia possibilita simular diferentes formas de intervenções na OAE e analisar quais destas intervenções possuem maior impacto na redução do nível de deterioração da construção, o que torna esse critério de avaliação bastante eficaz em um sistema de gestão de manutenções.

Contudo, percebe-se que há subjetividade por parte do profissional responsável pela inspeção, pois, os fatores de intensidade são definidos a partir de um referencial do Manual de aplicação da metodologia GDE/UnB em OAE's. Além disso, esse manual não contempla fatores relevantes para a vida útil de uma OAE, como é o caso da presença de erosão no aterro de encabeçamento do viaduto.

Nesse sentido, sugere-se pesquisas futuras com o objetivo de se identificar manifestações patológicas não contempladas no Manual de aplicação da metodologia GDE/UnB em OAE's, de forma a tornar cada vez mais eficiente a aplicação dessa metodologia na avaliação de obras de arte especiais. 


\section{AGRADECIMENTOS}

Agradecemos ao Centro Federal de Educação Tecnológica de Minas Gerais - CEFET-MG pelo apoio direto e indireto a este trabalho e ao Departamento de Edificações e Estradas de Rodagem de Minas Gerais - DEER-MG que autorizou a coleta de dados no viaduto da CMG-259, sobre a BR-135. Também agradecemos ao apoio do LAR - Laboratório de Avaliação e Reabilitação de Ambiente Construído do CEFET-MG - Campus Curvelo.

\section{REFERÊNCIAS}

ASSOCIAÇÃO BRASILEIRA DE NORMAS TÉCNICAS (ABNT). NBR 6118: Projeto de estruturas de concreto Procedimento. Rio de Janeiro: ABNT, 2014.

BOLDO, P. Avaliação quantitativa de estruturas de concreto armado de edificações no âmbito do Exército Brasileiro. Dissertação de Mestrado, Departamento de Engenharia Civil e Ambiental, Universidade de Brasília, Brasília, DF, 2002.

CASTRO, E. K. Desenvolvimento de metodologia para manutenção de estruturas de concreto armado, Dissertação de Mestrado, Departamento de Engenharia Civil, Universidade de Brasília, Brasília, DF, 1994.

EUQUERES, P. Metodologia de inspeção de pontes de concreto armado, Dissertação de Mestrado, Escola de Engenharia Civil, Universidade Federal de Goiás, Goiânia, GO, 2011.

FONSECA, R. P. A estrutura do Instituto Central de Ciências: Aspectos históricos, científicos e tecnológicos de projeto, execução, intervenções e propostas de manutenção. Dissertação de Mestrado em Estruturas e Construção Civil, Departamento de Engenharia Civil e Ambiental, Universidade de Brasília, Brasília, DF, 2007.

GiOVannetti, A. C. V. P. Avaliação do Estado de Conservação de Pontes - Estudo de Caso. Dissertação (Mestrado) - Curso de Engenharia Civil, Universidade Federal de Santa Catarina, Florianópolis, 2014.

GOMES, M. E. A.; XAVIER, T. J. B.; FRANCISCO, R. A. Levantamento e Cadastramento de Patologias Estruturais utilizando fotografias termográficas e drones na composição de banco geográficos. Projeto de Iniciação Científica - Curso de Engenharia Civil, Centro Universitário de Brasília, Brasília, 2018. Disponível em: <https://www.publicacoes.uniceub.br/pic/article/view/5774/4037>. Acesso em: 02 set. 2019. LOPES, B. A. R. Sistema de manutenção predial para grades estoques de edifícios: Estudo para inclusão do componente "Estrutura de Concreto". Dissertação de Mestrado, Departamento de Engenharia Civil, Universidade de Brasília, Brasília, DF, 1998.

MITRE, M. P. Metodologia para Inspeção e Diagnóstico de Pontes e Viadutos de Concreto. Dissertação (Mestrado em Engenharia Civil), Escola Politécnica da Universidade de São Paulo, São Paulo, 2005.

VERLY, R. C. Avaliação de Metodologias de Inspeção como Instrumento de Priorização de Intervenções em Obras de Arte Especiais. Dissertação (Mestrado em Engenharia de Estruturas), Departamento de Engenharia Civil, Universidade de Brasília, Brasília, 2015.

VITORIO, J. A. Um Estudo Comparativo sobre Métodos de Alargamento de Pontes Rodoviárias de Concreto Armado, com a utilização das normas brasileiras e Eurocódigos. Tese (Doutorado em Engenharia Civil), Universidade do Porto, Porto, 2013. 